\title{
Planificando el desarrollo CON IMPULSO DE LA UNIVERSIDAD - EMPRESA A TRAVÉS DE LA INNOVACIÓN Y LA TECNOLOGÍA
}

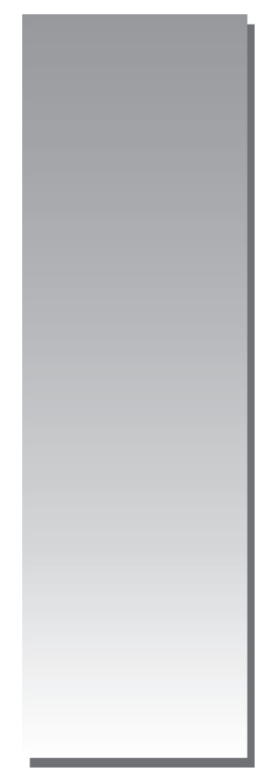

Planning Development with momentum of the University - COMPANY THROUGH INNOVATION AND teGhNOLOGY

\author{
Pablo Mauricio Pachas* \\ pwmauricio@yahoo.es
}

[RECEPCIÓN: ABRIL 2016 / CONFORMIDAD: MAYO 2016]

\begin{abstract}
RESUMEN
Objetivo: Identificar la calidad de la relación universidad - empresa en la transferencia de innovación y tecnología. Material y métodos: Estudio cualitativo de diseño descriptivo correlacional. Se revisaron modelos de innovación en relación a rankings del panorama universitario, tabulaciones sobre la investigación, inserción laboral e iniciativas de innovación en las empresas. Resultados: Se evidenció la relación directa entre la innovación aplicada por las empresas y la transferencia de tecnología, así como la productividad y el capital humano que ofertan las universidades. Esta relación contiene grandes potencialidades en beneficio del desarrollo socio- económico. Conclusiones: El fortalecimiento del binomio universidad-empresa, genera un desarrollo transversal tanto como en el desarrollo de tecnología, investigación y producción de conocimiento científico de carácter innovador. El adecuado fomento de este vínculo, conlleva a la creación de valor en las empresas, instituciones, mejorando las actividades productivas y elevando la capacidad del recurso humano por parte de las Universidades.
\end{abstract}

Palabras clave: Innovación; tecnología; empresa y desarrollo.

\section{ABSTRACT}

Objective: To identify the quality of the university - company relationship in the transfer of innovation and technology. Material and methods: Qualitative study of descriptive correlational design. Innovation models were reviewed in relation to university rankings, tabulations on research, labor insertion and innovation initiatives in companies. Results: The direct relationship between the

\footnotetext{
Economista. Magíster en Economía. Doctor en Ciencias Contables y Empresariales por la UNMSM. Posgrado en Finanzas, Proyectos, Bolsa de Valores en Instituciones Nacionales e Internacionales. Ha sido director general de planificación y presupuesto, de administración y asesor de alta dirección en el sector público, gerente de financieras, mutual y sociedad agente de bolsa. Consultor del Programa de Naciones Unidas para el Desarrollo (PNUD). Profesor visitante de las universidades PACE, New York y Columbia en el Estado de New York; Yale en New Haven, Connecticut; Northeastern, Boston y Suffolk en Boston, EE. UU. Becado por el Departamento de Estado de los Estados Unidos de América - International Visitor Leadership Program (2007). Ex Director de Posgrado FCA y ex Director de Negocios Internacionales de la Facultad de Ciencias Administrativas de la UNMSM. Actual Consultor (CGM). Profesor Principal de la UNMSM, profesor de Maestría y Doctorado en UNMSM, UNI, UNSA, U. Tacna etc. Expositor en la CCL y en diversas organizaciones.
} 
innovation applied by the companies and the transfer of technology, as well as the productivity and human capital offered by the universities, was evidenced. This relationship contains great potential for the benefit of socio-economic development. Conclusions: The strengthening of the universitycompany binomial, generates a transversal development as well as in the development of technology, research and production of innovative scientific knowledge. The proper promotion of this link, leads to the creation of value in companies, institutions, improving productive activities and increasing the capacity of human resources by universities.

Keywords: Innovation; technology; and development company.

\section{INTRODUCCIÓN}

A lo largo del tiempo, en el Perú se ha venido perdiendo la conexión innata que debe existir entre la universidad y la empresa para alcanzar su desarrollo, particularmente las universidades públicas se han venido alejando de su rol activo de aportar ciencias y tecnologías a la economía en su conjunto e impulsar el bienestar de la sociedad. Por ello, la intención de este trabajo es destacar la importancia del vínculo que debe existir entre la universidad y la empresa, para que sean aprovechados en un $100 \%$ la transferencia de ciencia, tecnología e innovación.

La imperante globalización ha venido destacando la necesidad que tienen todas las economías del mundo por la ciencia, la tecnología y la innovación; e impone un papel importante en el estudio de las dinámicas de innovación y tecnología que deben ser atendidas por las universidades y los centros tecnológicos. A esta tendencia, en el campo de la investigación existen evidencias acerca de la importancia que tienen los procesos de innovación en las interacciones entre universidadempresa-Estado o universidad pública-empresa.

En el actual contexto, se destaca la necesidad de las relaciones de cooperación entre las universidades y el sector empresarial, que cada vez más son entendidas como mecanismo de estímulo para el desarrollo de las ciencias, la tecnología y la innovación. En los últimos años se ha experimentado un importante proceso de cambio y modernización que ha supuesto una mayor interrelación con los países del entorno, en el que el sector privado externo e interno ha jugado un papel relevante para dinamizar la economía e incrementar los procesos de innovación empresarial.

La universidad, frente a las necesidades de innovación y tecnología que demandan las empresas y la economía en su conjunto para alcanzar el progreso, plantea el compromiso de desarrollar la investigación y la tecnología como aporte a la sociedad; estableciéndose como problema, ¿existe la conexión efectiva entre la universidad pública y la empresa?, ¿aporta ciencias y tecnologías a la economía en su conjunto e impulsa el bienestar de la sociedad?

\section{INVESTIGACIÓN Y DESARROLLO}

Respecto a la investigación y desarrollo $(I+D)$ comprende tres actividades fundamentales: investigación básica, investigación aplicada y desarrollo experimental. La investigación básica hace referencia a los trabajos experimentales o teóricos cuyo objetivo es obtener nuevos conocimientos acerca de los fundamentos de los fenómenos y hechos observables, sin necesidad de darles ninguna aplicación o utilización determinada. La investigación aplicada consiste también en trabajos originales realizados para obtener nuevos conocimientos, pero dirigido a un objetivo práctico específico. El desarrollo experimental se refiere a los trabajos sistemáticos que aprovechan los conocimientos obtenidos de la investigación o experiencia práctica para la producción de nuevos productos o dispositivos, así como a la puesta en marcha de nuevos procesos, sistemas y servicios, o a la mejora sustancial de los ya existentes (OECD, 2003)."

Con relación a la innovación existen numerosas definiciones, pero todas ellas coinciden en la idea de cambio, de algo nuevo que se lleva a cabo, aunque la diferencia está en delimitar qué es lo que cambia. La Real Academia de la Lengua Española define innovar como "mudar o alterar las cosas, introduciendo novedades". En esta misma fuente, innovación se define como "1. Acción y

\footnotetext{
OECD. (2003). Annual Report. enero 21, 2015, de THE ORGANISATION FOR ECONOMIC CO-OPERATION AND DEVELOPMENT (OECD) ANNUAL REPORT Sitio web: http:// www.oecd.org/about/2506789.pdf
} 
efecto de innovar; 2 . Creación o modificación de un producto, y su introducción en un mercado". En la actualidad, innovación se utiliza en distintas áreas del conocimiento ya sea económico, financiero, administrativo, social, entre otros.

En lo económico, la innovación está relacionada a la transacción comercial de un nuevo producto o proceso, cuya fase inicial corresponde a la invención y la fase final es la aplicación. En ambas fases existe un amplio proceso que puede llevar al fracaso la aplicación de la innovación. La innovación conlleva un elevado riesgo, por tanto apreciable retorno de la inversión. En este aspecto contribuyó el economista Schumpeter, quien estableció claramente la diferencia entre los conceptos de "innovación" e "invento" como una idea para la consecución de un producto, proceso o sistema nuevo o mejorado, pero que aún no ha sido aplicado (innovación). Este mismo autor atribuyó un papel destacado dentro del proceso de innovación a los empresarios, como actores implicados directamente en la "transformación" de invento a innovación (Schumpeter, 1934)" .

Según Drucker (1988) acción de dotar a los recursos con una nueva capacidad de producir riqueza, así como también dice que cualquier cambio en el potencial productor de riqueza ya existente. Innovación es la producción de un nuevo conocimiento tecnológico, diferente de la invención que es la creación de alguna idea científica teórica o concepto que pueda conducir a la innovación cuando se aplica en el proceso de producción (Elster, 1990)

La innovación es la herramienta específica de los empresarios innovadores, el medio con el cual explotar el cambio como una oportunidad para un negocio diferente; el empresario innovador ve al cambio como norma saludable. No necesariamente lleva el cambio él mismo, pero busca el cambio, responde a él y lo explota como una oportunidad (Druker, 1988).

Los trabajos de Schumpeter (1934) han influido notablemente en las teorías de la innovación. Afirmaba que el desarrollo económico está movido por la innovación, por medio de un proceso dinámico en el cual nuevas tecnologías sustituyen

\footnotetext{
*** Schumpeter (1934) . La teoría de la económica desarrollo: Una investigación sobre los beneficios, Capital, Crédito, el interés y el ciclo económico. Transacción Editores.

***** Drucker (1988). Llegada de la nueva organización

******** Elster, J. (1990). "Norms of revenge". En Ethics, vol. 100, pp. 862-85.
}

a las antiguas, denominando a este proceso "destrucción creativa". Las innovaciones "radicales" originan los grandes cambios del mundo, mientras que las innovaciones "progresivas" alimentan de manera continua el proceso de cambio. Schumpeter propuso cinco tipos de innovaciones: a) introducción de nuevos productos; b) introducción de nuevos métodos de producción; c) apertura de nuevos mercados; d) desarrollo de nuevas fuentes de suministro de materias primas u otros insumos; y e) creación de nuevas estructuras de mercado en un sector de actividad.

El proceso de innovación puede concebirse como la actividad racional dirigida a una meta o, de otro lado, como un proceso de ensayo y error, resultado de la suma acumulativa de ciertas modificaciones del proceso de producción, pequeñas y en gran medida accidentales (Elster, 1990). La concepción de proceso de innovación varía de acuerdo a diferentes perspectivas y escuelas de pensamiento económico. Se trata de la introducción de un nuevo producto, bien o servicio, significativamente mejorado, de un proceso, de un nuevo método de comercialización o de un nuevo método organizativo en las prácticas internas de la empresa, la organización del lugar de trabajo o las relaciones exteriores.

Las actividades innovadoras incluyen todas las actuaciones científicas, tecnológicas, organizativas, financieras y comerciales que conducen a la introducción de innovaciones. Se distinguen cuatro tipos de innovación: a) Innovación de producto, aporta un bien o servicio nuevo, o significativamente mejorado, en cuanto a sus características técnicas o al uso al que se destina. Incluye la mejora significativa de las características técnicas, de los componentes y los materiales, de la informática integrada, de la facilidad de uso u otras características funcionales. b) Innovación de proceso es la introducción de un nuevo o significativamente mejorado proceso de producción o distribución. Se aplican cambios significativos en las técnicas, los materiales o los programas informáticos. Las innovaciones de proceso pueden tener por objeto la disminución de los costos unitarios de producción o distribución, mejorar la calidad, la producción o distribución de productos nuevos o sensiblemente mejorados. Los métodos de producción incluyen técnicas, equipos y programas informáticos utilizados para producir bienes o servicios. Los métodos de distribución están vinculados a la logística de la empresa y 
engloban los equipos, los programas informáticos y las técnicas para el abastecimiento de insumos, la asignación de suministros en el seno de la empresa o la distribución de productos finales. c) Innovación en marketing es la aplicación de un nuevo método de comercialización que implique cambios significativos en el diseño, envasado, posicionamiento, promoción o tarifas. Las innovaciones en marketing tratan de satisfacer mejor las necesidades de los consumidores, de abrir nuevos mercados, de posicionar de una nueva manera un producto con el objeto de aumentar las ventas. d) Innovación en organización es la introducción de un nuevo método organizativo en las prácticas, la organización del lugar de trabajo o las relaciones exteriores de la empresa. Su objetivo es mejorar los resultados reduciendo costos administrativos o de transacción, mejorando el nivel de satisfacción del trabajo, facilitando el acceso a conocimiento o reduciendo los costos de suministro.

Respecto a transferencia de tecnología, de modo amplio, se trata de la transferencia de conocimiento o tecnología entre entidades a través de diferentes medios, herramientas contractuales o de acuerdo entre las partes. En las transacciones de tecnología se hace una diferencia entre tecnologías inmateriales y materiales. Las inmateriales comprenden la adquisición de tecnología bajo la forma de patente, invenciones no patentadas, licencias, informes de know-how, marcas de fábrica, diseños, modelos de utilidad, compra de servicios de investigación y otros servicios de contenido tecnológico. Por tecnologías materiales se entiende la adquisición de equipos o maquinarias con un contenido tecnológico relacionado con las innovaciones de productos y procesos introducidos por la organización.

La teoría neoclásica establece a los procesos de innovación como una tarea exclusiva de los centros de investigación exógenos a las empresas, y por lo tanto exógeno también al proceso de producción y a los esfuerzos de maximización de los beneficios. En su intento por establecer modelos con una gran capacidad predictiva, establece la información como un bien público al cual todo mundo puede acceder de manera libre y gratuita, y por lo tanto, los costos de las transferencias tecnológicas y del aprendizaje son mínimos para la sociedad. La teoría ortodoxa se construye sobre el supuesto metodológico de que los actores toman decisiones "como....si" su objetivo exclusivo fuera el maximizar el beneficio o la utilidad (Friedman, M. 1986 en Lara, A. 2007)

A diferencia de la teoría neoclásica, la teoría evolutiva viene a sustituir al agente neoclásico por un agente con capacidades limitadas el cual busca "satisfacer" necesidades de adaptación y supervivencia. La teoría evolutiva tiene su origen en los trabajos de Joseph Shumpeter y una de las tesis centrales de esta teoría sostiene que para captar la naturaleza de la conducta de los agentes es necesario poner en el centro del problema la adaptación y la práctica que ella trae aparejada (Lara, A. 2007). Uno de los principales problemas que se plantea esta teoría no es cómo el modo de producción capitalista administra las estructuras existentes, sino cómo las crea y las destruye (origen del concepto "destrucción creadora"), por lo que los agentes se enfrentan al reto de la supervivencia. A diferencia también de la teoría neoclásica, la teoría evolutiva concibe a la información como un bien privado el cual se genera y se enfrenta a un proceso de apropiación, por lo que el desarrollo tecnológico se da de manera distinta entre los diversos agentes.

La teoría evolutiva viene a constituir las bases fundamentales que han generado el desarrollo de nuevos enfoques y ha proporcionado las herramientas analíticas para la explicación de nuevos fenómenos para los cuales la teoría ortodoxa presenta modelos ineficientes. La evolución histórica de las teorías sobre los procesos de innovación y de la cual se desprende el enfoque de los sistemas de innovación, tiene su antecedente principal en la teoría evolutiva antes mencionada y ha pasado desde su concepción por varias etapas, variando la forma en que el proceso es asimilado por el medio económico. La discusión de las dos corrientes principales de los procesos de innovación podemos encontrarla en la década de los setenta, el modelo basado en la teoría neoclásica de la innovación posee un carácter lineal donde la importancia radica en identificar los factores determinantes de los resultados de la investigación tecnológica. Este modelo concibe a la innovación como un proceso que pasa por distintas etapas: investigación, invención, innovación y difusión; y, de otro lado, la transferencia de conocimientos se describe como un proceso natural y no se le asigna un costo significativo dentro del proceso.

Urbina E. (2010). "Proceso de innovación”. Enero 25, 2015, de Tesis Uson. Sitio web: <http://tesis.uson.mx/ digital/tesis/docs/19264/Capitulo1.pdf > . 
Figura 1: Modelo lineal de innovación

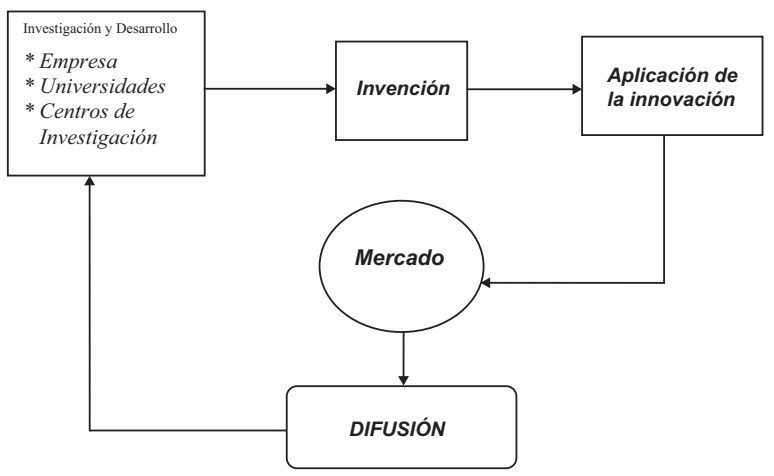

Una de las principales limitaciones de esta teoría es que tiende a subestimar la actividad innovadora de las pequeñas empresas, pues un indicador de innovación básico de estos agentes económicos es la inversión en investigación y desarrollo (I+D). Bajo el enfoque de la teoría evolutiva, a inicio de los años ochenta se desarrolló el modelo interactivo de la innovación, el cual se basa en la idea de una interacción continua entre los distintos partícipes y elementos presentes durante todo el proceso de innovación y la comercialización posterior, considerando los ambientes de innovación, las redes interinstitucionales y las rutinas generadas dentro de las empresas como factores fundamentales de este proceso. Este nuevo modelo implica cambios radicales para la gestión tecnológica de las empresas y el diseño de la política tecnológica por parte de las administraciones gubernamentales.
Figura 2: Modelo interactivo de innovación

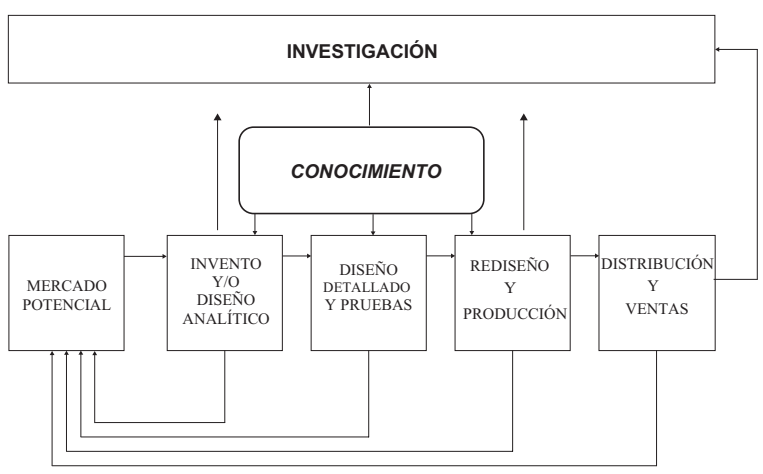

Los cambios que se dan en el mercado y en la tecnología, incluyendo los avances de los propios competidores, los obligan a participar y seguir en la carrera para adaptarse a los cambios. En la Teoría económica de la innovación industrial, C. Freeman, (1974) trata las diferentes estrategias que puede adoptar una empresa ante la innovación. Entre estas tenemos a la estrategia innovadora ofensiva, la innovadora defensiva, la imitativa, la dependiente, la tradicional y la oportunista o de nicho. Según el enfoque de los sistemas de innovación, estas se presentan de manera articulada en cuatro niveles y cada uno adquiere una dinámica propia y que difiere con las otras, además de ofrecer un marco particular para la elaboración y aplicación de una política económica.

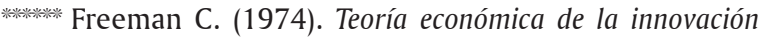
industrial. Madrid: Editorial Jus.

Figura 3. Sistema de innovación y sus relaciones

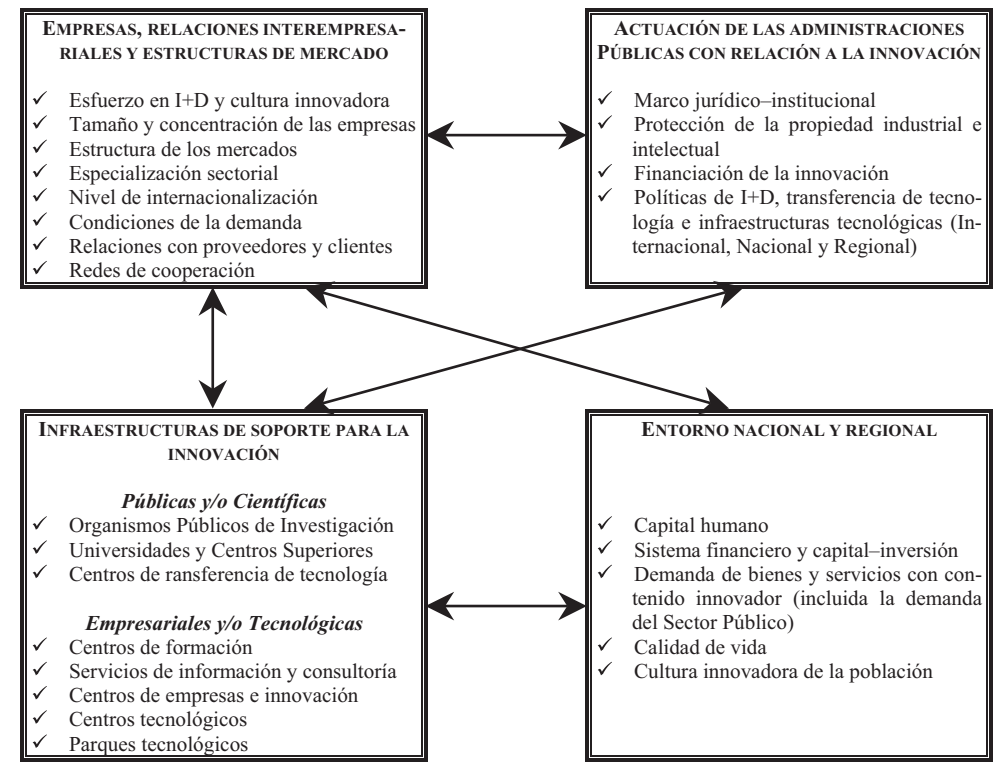


En primer término, este enfoque visualiza a la empresa y las relaciones interempresariales como el ente contenedor de conocimientos que están en sus rutinas operacionales y se van modificando con el paso del tiempo gracias a procesos de investigación, reglas de conducta, decisiones estratégicas, etc. En segundo término, la empresa se va vinculando con otras empresas e instituciones, como lo son las universidades, los centros de investigación, etc.; vinculación que origina una red de cooperación e integración donde la actuación de la administración pública es fundamental para sentar las bases de un entorno favorable para el desarrollo tecnológico.

En un ámbito más amplio, se establecen un conjunto de factores como las relaciones sociales y la cultura innovadora, este último ámbito abarca principalmente la capacidad de absorción de tecnología. De manera que el enfoque de los sistemas de innovación se puede establecer como un enfoque "holístico" que sitúa en el centro del análisis a la innovación y trata de analizar todos los factores que la determinan, e interdisciplinario al tomar en cuenta tanto factores económicos como sociales, políticos, entre otros.

\section{INTERRELACIÓN UNIVERSIDAD-EMPRESA}

El presente trabajo trata la interrelación universidad-empresa, destacando las necesidades que tienen las empresas por la innovación y la tecnología así como su vínculo con la universidad, frente a la imperante globalización y los cambios que se vienen experimentando en el contexto económico y financiero. Estas dos organizaciones son de mucha importancia para el crecimiento económico y bienestar de la sociedad. La situación económica actual revela la necesaria interrelación universidad-empresa, particularmente las universidades públicas que son financiadas con recursos públicos y se deben a toda la sociedad, por tanto tienen como compromiso aportar ciencia y tecnología, además de contribuir con elevar la productividad y el bienestar de toda la sociedad peruana.

El estado pasivo de la universidad en el Perú debe cambiar y responder a los retos y desafíos que exige el actual contexto económico y social. Se debe prestar atención a las revelaciones de que la universidad peruana no se encuentra entre las diez mejores universidades de Latinoamérica, según la consultora británica Quacquarelli Sy- monds - QS de las universidades de Latinoamérica - 2015. En el primer puesto se ubica la Universidad de Sao Paulo del Brasil, lo que resulta paradójico que siendo el Perú el segundo país con mayor número de universidades 140, después de Brasil que alcanza a 197, con una población de 202 millones mientras que nuestro país tiene 30 '814.175, no figure entre las mejores de Latinoamérica.

Cuadro 1: Ranking de las diez mejores universidades de Latinoamérica 2015

\begin{tabular}{cccc}
\hline Puesto & Puntaje & Universidad & País \\
\hline 1 & 100 & $\begin{array}{c}\text { Universidad de Sao Paulo } \\
2\end{array}$ & Brasil \\
\hline 3 & 97.4 & $\begin{array}{c}\text { UniversidadEstadualdeCam- } \\
\text { pinas (Unicamp) }\end{array}$ & Brasil \\
\hline 4 & 95.9 & PontificiaUniversidaddeChile & Chile \\
\hline 5 & 95.1 & $\begin{array}{c}\text { Universidadad de Chile } \\
\text { Janeiro }\end{array}$ & Chile \\
\hline 6 & 94.9 & $\begin{array}{c}\text { NacionalAutónomadeMéxico } \\
\text { (UNAM) }\end{array}$ & México \\
\hline 7 & 94.4 & Universidad de Los Andes & Colombia \\
\hline 8 & 90 & UniversidadEstadualPaulista & Brasil \\
\hline 9 & 86.7 & Tecnológico de Monterrey & México \\
\hline 10 & 85 & Universidad de Brasilia & Brasil \\
\hline
\end{tabular}

En el caso de universidades peruanas, la Universidad Católica (PUCP) se ubica en el puesto 19, mientras que San Marcos (UNMSM) ocupa el puesto 60 .

Cuadro 2: Ranking de las universidades del Perú 2015

\begin{tabular}{|c|c|c|c|}
\hline Puesto & Puntaje & Universidad & País \\
\hline 19 & 76.7 & $\begin{array}{c}\text { PontificiaUniversidadCatólica } \\
\text { del Perú (PUCP) }\end{array}$ & Perú \\
\hline 60 & 54.7 & $\begin{array}{l}\text { UniversidadNacionalMayorde } \\
\text { San Marcos }\end{array}$ & Perú \\
\hline 64 & 53.6 & UniversidadCayetanoHeredia & Perú \\
\hline 102 & 39.9 & Universidad de Lima & Perú \\
\hline 117 & 36.7 & $\begin{array}{l}\text { Universidad Nacional Agraria } \\
\text { La Molina }\end{array}$ & Perú \\
\hline 134 & 34.5 & $\begin{array}{c}\text { UniversidadNacionaldelnge- } \\
\text { niería }\end{array}$ & Perú \\
\hline 139 & 35.3 & Universidad del Pacífico & Perú \\
\hline
\end{tabular}

Consultora QS. (2015). "QS University Rankings: Latin America 2015”. enero13, 2015, de Topuniversities Sitio web: <http://www.topuniversities.com/ university-rankings/latam-university-rankings/2015 $\#$ sorting $=$ rank + region $=+$ country $=+$ faculty $=+\mathrm{s}$ tars $=$ false + search $=>$. 
Situación que conlleva elevar el esfuerzo por fijar objetivos basado en una construcción de visión de futuro y asumir firmes compromisos en el corto plazo ejecutando metas articuladas a objetivos estratégicos de largo plazo.

Hay opiniones encontradas, como las de la Confiep frente a la nueva ley universitaria que se mostró en contra, que si bien no es la solución a toda la problemática universitaria, sí introduce cambios en la gestión, y establece orientaciones para la mejora de la calidad académica. De otro lado, la Confiep reconoce que en el Perú existe una falta de capital humano y cuestiona la calidad académica en la formación profesional por parte de las universidades públicas, por lo que requiere cambios.

La utilización de las tecnologías informáticas y de comunicaciones ha crecido considerablemente, y las empresas dependen cada vez más de ellas para responder a las exigencias del entorno empresarial actual y competitividad. La volatilidad de los mercados y la incertidumbre que hay que atender, hacen que la Gestión de la Tecnología y la Innovación (GTI) sea uno de los factores clave para el logro del éxito de las organizaciones empresariales, sean grandes o pequeñas. La habilidad que tenga la organización para implantar y mantener las tecnologías modernas de manera eficiente y controlada, tendrá un gran impacto en su grado de competitividad y optimización de los recursos. Los mercados se tornan muy competitivos y para poder insertarse en ellos es necesario la constante innovación e investigación e impulsar el desarrollo.

Se ha observado que el crecimiento económico experimentado en el país en los últimos años ha venido acompañado de una creciente actividad en investigación e innovación, explicando la necesidad de fortalecer la relación de las instituciones del Estado, academia y empresa, y propiciar el desarrollo del país. La mayor actividad económica y empresarial se produce a partir de la década del noventa, en que se supera la recesión económica (ver círculo del cuadro) y la inestabilidad del mercado (hiperinflación), y se inicia la senda del crecimiento, estabilidad del mercado y mayores inversiones en el sector real acompañado de programas de investigación, desarrollo y tecnologías. Tal recesión profundizada entre 1988, 1989 y 1990 , y el crecimiento a partir del 1991 al 2014 se pueden apreciar en el siguiente cuadro:
Cuadro 3: Evolución del PBI (\%), 1985-2014.

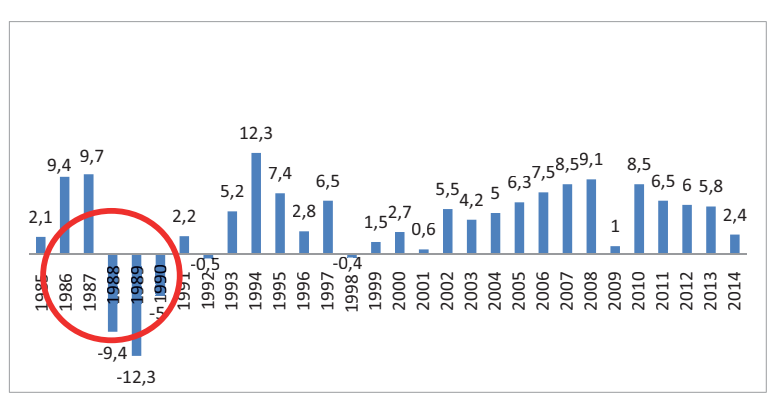

Fuente: INEI.

Elaboración propia

Tal trayectoria de crecimiento ha sido sostenida desde 1993 a la fecha (2015), aun cuando la tasa de crecimiento disminuye en 1996, 1999 -2001, y desde el 2011 al 2015 siempre se incrementa el PBI, impulso que se debe al aumento de las inversiones y a la vigencia de la apertura económica y comercial puesta en marcha a inicio de los noventa. Tal crecimiento sostenido desde 1993 se puede apreciar en el siguiente cuadro:

Cuadro 4: Crecimiento sostenido del PBI (Miles de soles), $1993-2013$.

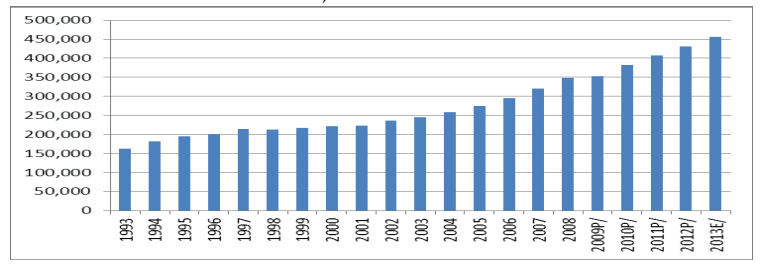

Fuente: INEI

Por lo que resulta importante involucrar procesos de innovación y tecnología para gestionar con eficiencia las actividades y procesos de las empresas. Ante la imperante globalización se tienen que fortalecer las capacidades y herramientas para innovar y competir en los mercados internacionales, y no hay duda que se debe mejorar la competitividad en todos los aspectos, por lo que resulta imperativo el enlace universidad pública y empresa. Es una preocupación que hay que atender dado el constante desarrollo tecnológico que ha tenido lugar en las últimas décadas del siglo XX y en el presente siglo, donde el ciclo de vida de un producto o tecnología es fugaz y se hace necesaria una constante renovación para no perecer en un mercado cada vez más competitivo.

Del análisis se pueden establecer los resultados y discusión cómo el enlace entre la universidad pública y empresa, mediante la innovación y tec- 
nología, impulsará el avance de la transferencia de tecnología de la universidad pública a la economía del país. Microsoft encargó un estudio a nivel mundial a la consultora Ipsos, el cual incluyó al Perú entre los países analizados; estudio que reveló como conclusión principal que existe una relación directa entre el uso de la tecnología y el crecimiento comercial de las empresas que lo adoptan.

Así, las pymes que hacen uso intensivo de la tecnología crecieron $18 \%$ en su facturación, frente a un $10 \%$ en las empresas que hacen menor uso (o ninguno) de las TIC, durante el período 20112013. Asimismo, el $57 \%$ de las pymes con uso intensivo de tecnología resaltaron su valor para la productividad de su negocio, frente a un $43 \%$ de las empresas que no adoptaron mucho la tecnología. De acuerdo al estudio, cada vez las empresas emplean más tecnología y la más usada por las pymes es la cuenta o página en redes sociales de la empresa. También cabe destacar que hace tres años el $35 \%$ de las empresas encuestadas utilizaba algún servicio de computación en la nube, hoy ha aumentado a $42 \%$. El crecimiento de los servicios tecnológicos utilizados por las empresas se puede apreciar en el siguiente cuadro:
Si bien se destaca el rol de la universidad frente a la empresa y la importancia de la tecnología e innovación para actualizarse a los tiempos actuales, se advierte que hay débil respuesta de las universidades públicas por la competitividad del profesional egresado. Si las universidades son las que preparan el talento humano que necesitan las empresas, ¿̇por qué no responden a las exigencias del mercado?, de igual manera podríamos preguntarnos ¿están las universidades totalmente abiertas a las tendencias laborales?

Se aprecia de manera significativa que los perfiles de las carreras profesionales no responden a las necesidades de cambios que experimentan las empresas en el país, ya sean grandes o pequeñas se desenvuelven en mercados altamente competitivos, y que al no haber en el mercado laboral interno tengan que proveerse de recursos humanos extranjeros. Por lo que resulta principal preocupación de las universidades brindar una formación profesional de calidad orientada a satisfacer las demandas laborales de las empresas. Según el INEI, en su encuesta nacional describe a las universidades y su tasa de ocupación de sus egresados, donde destacan las privadas.

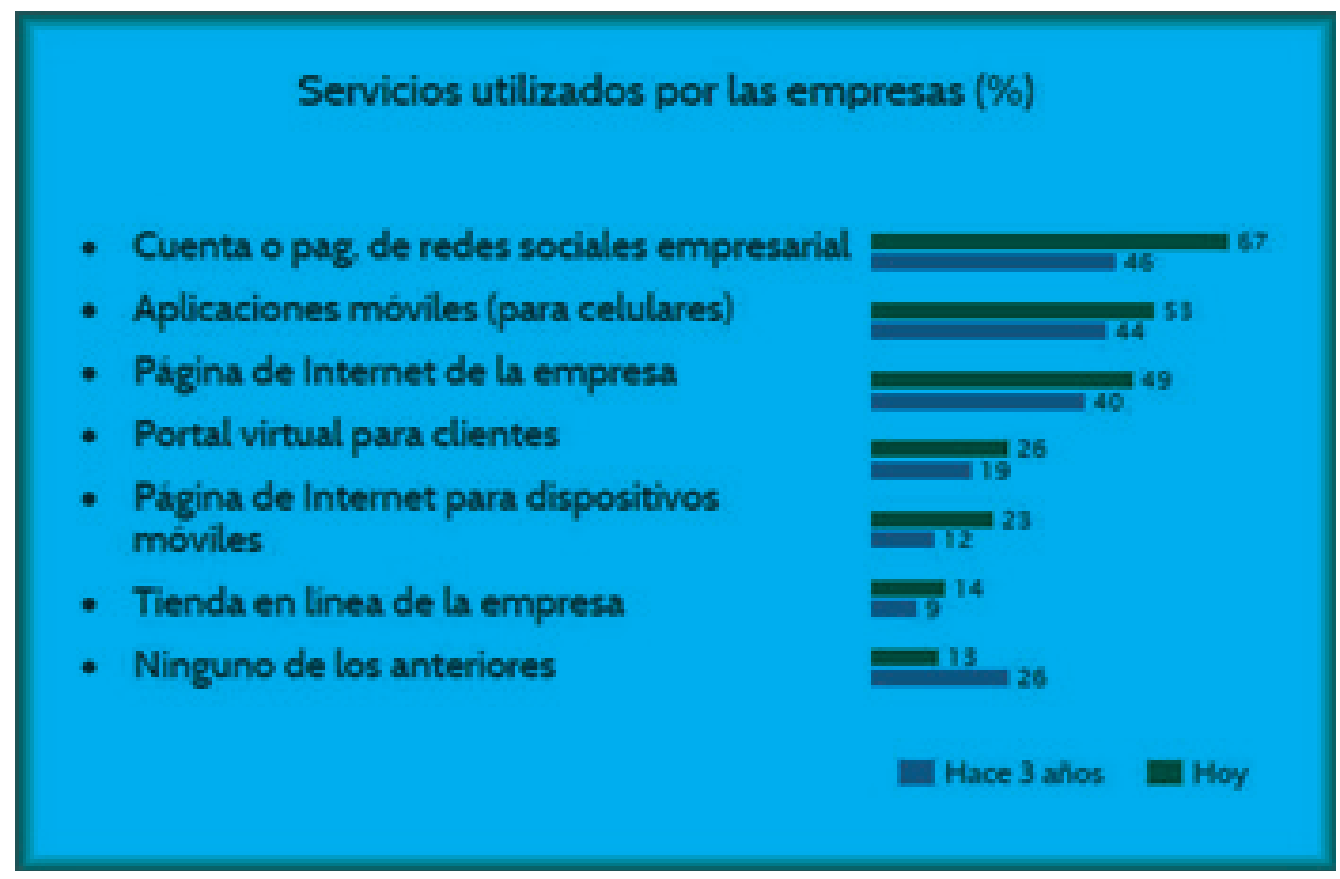




\begin{tabular}{|c|c|c|c|}
\hline \multirow{2}{*}{ Nombre de universidad } & \multirow{2}{*}{ Total } & \multicolumn{2}{|c|}{ Mayores niveles de empleo } \\
\hline & & Abs. & $\%$ \\
\hline Universidad Nacional de San Martín & 1929 & 1828 & 94,8 \\
\hline Universidad Peruana de Ciencias Aplicadas & 3453 & 3269 & 94,7 \\
\hline Universidad del Pacífico & 1043 & 987 & 94,6 \\
\hline Universidad Nacional Mayor de San Marcos & 8593 & 7979 & 92,9 \\
\hline Universidad Nacional del Centro Del Perú & 3367 & 3056 & 90,8 \\
\hline Universidad Nacional de San Antonio Abad del Cusco & 4283 & 3881 & 90,6 \\
\hline Universidad Nacional del Callao & 3692 & 3337 & 90,4 \\
\hline Universidad de San Martin de Porres & 9893 & 8939 & 90,4 \\
\hline Universidad Privada San Pedro & 3481 & 3145 & 90,3 \\
\hline Universidad Andina del Cusco & 3484 & 3139 & 90,1 \\
\hline Universidad Nacional de Ingenieria & 3199 & 2879 & 90,0 \\
\hline Universidad Peruana Los Andes & 3016 & 2713 & 90,0 \\
\hline Universidad Privada Antenor Orrego & 2490 & 2241 & 90,0 \\
\hline Universidad Católica los Ángeles de Chimbote & 3689 & 3300 & 89,5 \\
\hline Pontificia Universidad Católica del Perú & 6388 & 5702 & 89,3 \\
\hline
\end{tabular}

Fuente: Instituto Nacional de Estadística e Informática - INEI - Encuesta Nacional a Egresados Universitarios y Universidades, 2014

Al concluir los estudios universitarios surge como natural preocupación en los egresados tener un empleo. Ante la mayor de oferta de egresados universitarios las empresas elevan sus requerimientos y prefieren los titulados que avalarían puestos de mayor responsabilidad. Según el INEI, en su encuesta nacional describe que el $45,9 \%$ de los egresados universitarios ocupados cuentan con el título profesional o licenciatura, el 39,5\% cuentan con el grado de bachiller y el 14,6\% no tienen grado o simplemente es egresado.
Las diez carreras más demandadas el 2014 fueron: Administración de empresas, Contabilidad, Administración hotelera, Administración de negocios internacionales, Computación e Informática, Ingeniería industrial, Administración financiera, Economía, Administración industrial y Marketing. Según el portal trabajando.com.

De otro lado, las carreras con puestos mejor pagados son Administración de empresas, Ingeniería industrial, Ingeniería civil, Economía, Contabilidad, Administración industrial, Ingeniería

Cuadro 7: Egresado universitario -2014.

\begin{tabular}{lrr}
\hline \multicolumn{1}{c}{ Grado académico } & Abs. & $\%$ \\
\hline Total & 171715 & 100,0 \\
\hline Titulado o licenciado & 78864 & 45,9 \\
Bachiller & 67759 & 39,5 \\
Egresado & 25092 & 14,6 \\
\hline Fuente: Instituto Nacional de Estadistica e Informatica - INEI - Encuesta Nacional a Egresados Universitarios \\
y Universidades, 2014
\end{tabular}

Cuadro 8: Carreras más demandadas 2015

\begin{tabular}{|l|l|}
\hline \multicolumn{2}{|c|}{ LAS CARRERAS MÁS DEMANDADAS DURANTE EL 2015} \\
\hline $\mathbf{1}$ & Administración de Empresas \\
\hline $\mathbf{2}$ & Contabilidad \\
\hline $\mathbf{3}$ & Ingeniería Industrial \\
\hline $\mathbf{4}$ & Economía \\
\hline $\mathbf{5}$ & Administración de Empresas (Técnico) \\
\hline $\mathbf{6}$ & Ingeniería en Informática / Sistemas \\
\hline $\mathbf{7}$ & Computación e Informática \\
\hline $\mathbf{8}$ & Derecho \\
\hline $\mathbf{9}$ & Administración de Negocios Internacionales \\
\hline $\mathbf{1 0}$ & Ciencias de la Comunicación \\
\hline Fuente: Trabajando.com \\
\hline
\end{tabular}

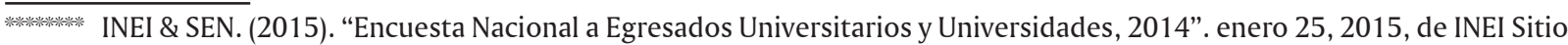
web: <https://www.inei.gob.pe/media/MenuRecursivo/publicaciones_digitales/Est/Lib1298/Libro.pdf> . 
mecánica, Derecho, Ingeniería mecánica-eléctrica e Ingeniería civil.

En el presente estudio se ha resuelto la hipótesis de la investigación como: "el enlace de la universidad y la empresa a través de la innovación y la tecnología eleva la competitividad del profesional egresado". Asimismo se responde a la hipótesis específica: "La débil respuesta de la universidad a las necesidades de tecnología que demanda la empresa, conlleva la deficiencia de los recursos aportado por toda la sociedad".

Los resultados establecen la necesidad de desarrollar la investigación en todas las áreas, en las universidades públicas y aprovechar el elevado número de talento humano para transferir ciencia y tecnología a las empresas y a la sociedad. Las universidades deben trabajar en la formación integral de sus profesionales, capacitarlos en el uso de herramientas tecnológicas necesarias para su desenvolvimiento profesional, motivarlos a innovar y convertirlos en las excelentes vitrinas que necesitan las empresas hoy en día, de ese modo se elevaría la competitividad del profesional egresado. Los empresarios reconocen un déficit de capital humano, no encuentran personal calificado para ciertos puestos de trabajo y tienen que tomarlos del exterior, lo que explica la ausencia de las universidades públicas en la actividad económica y social, dado que tienen la mayor población estudiantil universitaria.

La universidad como centro de investigación, innovación y tecnologías, se debe impulsar a través de redes de organizaciones que tienen fines de investigación y tecnologías y que establecen instrumentos de cooperación con el propósito de crear valor a los productos, sean bienes o servicios (UPCH-2012). Los profundos cambios tecnológicos, económicos y sociales son los nuevos retos que los centros de investigación, tecnológicos e innovación de las universidades deben asumir, para conectarse a los nuevos modelos de negocios y las formas de administrar y de gestionar las empresas, y de este modo generar ventajas competitivas sostenibles que permitan contribuir al desarrollo del país.

Se debe tener presente que las empresas son el centro de los sistemas de innovación, ya que ellos son los agentes principales encargados del traslado de resultados de investigación al mercado, así como la absorción de nuevas tecnologías y su posterior impacto en el incremento de la produc- tividad. En el Perú, las actividades productivas se concentran principalmente en servicios de baja complejidad tecnológica, industria extractiva, agricultura y manufactura con baja complejidad tecnológica; todo ello implica que la demanda de tecnología y conocimiento productivo sea reducida; a esto se suma que los niveles de inversión en $\mathrm{I}+\mathrm{D}$ son limitados y como resultado de ello la actividad innovadora es muy baja. De modo que el rol que tienen las universidades y centros de investigación es vital, ya que la generación de conocimientos puede ser aprovechado por el sector empresarial y facilitar la transferencia tecnológica fortaleciendo el proceso innovador.

Actualmente esta vinculación es mínima y poco frecuente, como se describe en la información proveniente de la Encuesta Económica Anual donde nos muestra que menos del $3 \%(2.8 \%)$ de empresas encuestadas tienen actividades conjuntas con institutos de investigación o universidades para la realización de proyectos de innovación o de mejoras tecnológicas para el desarrollo de nuevos productos. Si bien este porcentaje es mayor para empresas grandes, tampoco llega al $4 \%$ (3.6\%) de la totalidad de empresas (ver cuadro). La escasa vinculación también impacta negativamente en la alineación y direccionamiento de los temas de investigación, sobre todo aplicados con las necesidades del sector empresarial. Al no relacionarse ambos sectores es difícil conocer cuál es la demanda potencial de servicios de investigación y transferencia tecnológica, así como de las capacidades y oferta de los institutos de investigación ${ }^{-}$, tarea que debe ser atendida por la universidad.

Como se mencionó en el encuentro científico internacional, en todo país del mundo desarrollado y en vías de desarrollo, el principal centro de producción científica y creación de conocimientos es la universidad; por lo que existe una correlación positiva entre el nivel de desarrollo de un país y la calidad de su universidad. Después de la revisión de los indicadores de $\mathrm{C}+\mathrm{T}+\mathrm{I}$ y de la situación de la universidad en el Perú, es deber ineludible formular una propuesta que coadyuve a resolver esta problemática que tiene cerca de tres décadas sin solución.

\footnotetext{
料料料粠 CONCYTEC. (2014). Estrategia Nacional para el Desarrollo de la Ciencia, Tecnología e Innovación documento sujeto a consulta pública CREAR PARA CRECER. Enero 13, 2015, de sitio web: <http:// portal.concytec.gob.pe/images/stories/images2014/ mayo/crear_crecer/estrategias_crear_crecer_ultima version_28-5-2014.pdf $>$.

橉橉橉 Jaime $\bar{E}$. Luyo. (2012). "La ciencia, tecnología e innovación y la universidad peruana en el siglo XXI". Enero 14, 2015, de Competitiveness and Sustainable
} 
Cuadro 9: Número de empresas que realizaron proyectos de innovación o de mejoras tecnológicas para el desarrollo de nuevos productos* con institutos de investigación o universidades (abril 2010 - marzo 2011).

\begin{tabular}{|c|c|c|c|c|}
\hline \multirow{2}{*}{\multicolumn{2}{|c|}{ ESTRATO EMPRESARIAL }} & \multicolumn{3}{|c|}{$\begin{array}{c}\text { Actividades conjuntas con institutos de investigación o } \\
\text { universidades** }\end{array}$} \\
\hline & & \multirow{2}{*}{$\begin{array}{l}\text { Si } \\
34\end{array}$} & \multirow{2}{*}{$\begin{array}{c}\text { No } \\
1279\end{array}$} & \multirow{2}{*}{$\begin{array}{l}\text { Total } \\
1313\end{array}$} \\
\hline & Frecuencia & & & \\
\hline & $\%$ & 2.6 & 97.4 & 100.0 \\
\hline \multirow{2}{*}{ Mediana Empresa } & Frecuencia & 6 & 342 & 348 \\
\hline & $\%$ & 1.7 & 98.3 & 100.0 \\
\hline \multirow{2}{*}{ Gran Empresa } & Frecuencia & 31 & 837 & 868 \\
\hline & $\%$ & 3.6 & 96.4 & 100.0 \\
\hline \multirow{2}{*}{ Total } & Frecuencia & 71 & 2458 & 2529 \\
\hline & $\%$ & 2.8 & 97.2 & 100.0 \\
\hline
\end{tabular}

Cabe señalar que la universidad no solo debe estar orientada a la innovación, sino como se ha establecido al desarrollo de las ciencias y crear conocimiento de alta calidad, donde las universidades que realizan investigación no deberían limitarse a la creación de conocimiento, sino que debe buscar proyectar dicho conocimiento en la sociedad.

El acercamiento de las universidades del país a las empresas, particularmente las públicas, ha sido débil, de modo general han tratado de aportar mediante proyectos de investigación, es por ello que como en otras partes del mundo se vienen promoviendo programas que apoyan las iniciativas de negocio y la creatividad empresarial, en nuestro medio se describe una relación incipiente por tanto con mínimo impacto en la sociedad y en el crecimiento económico del país.

En nuestro país están surgiendo instituciones con la misión de promover la ciencia, la tecnología y la investigación. Uno de estos casos es Concytec, que anunció su nueva estrategia nacional para el desarrollo de la CTI (ciencia, tecnología e innovación). De parte del Estado se ha creado en el 2007 la Unidad Coordinadora del Programa de Ciencia y Tecnología (Fincyt)-PCM, orientado a contribuir al incremento de la competitividad del país, fortaleciendo las capacidades de investigación e innovación tecnológica y promoviendo la articulación de la empresa, universidad y Estado, mediante el otorgamiento de financiamiento, en estrecha coordinación con el Consejo Nacional

Development Institute en sitio web: <http://www. encuentrocientificointernacional.org/reportescienciap eru/201202febrero/201202jaimeluyouniversidad.pdf > . Albornoz M. \& López J. (2010). "Ciencia, tecnología y universidad en Iberoamérica". Enero 14, 2015, de Metas Educativas 2021 en sitio web: <http://www. oei.es/salactsi/ciencia_universidades.pdf $>$. de Ciencia, Tecnología e Innovación Tecnológica (Concytec) y el Consejo Nacional de Competitividad (CNC).

El Fincyt en su balance describe que del total de proyectos recibidos de las universidades, pocos han sido aprobados. De los 725 proyectos de investigación en el período 2007-2010, 305 proyectos provienen de las universidades y centros de investigación y 420 de las empresas, solo 177 fueron aprobados. Esta institución estimula la alianza entre la universidad y la empresa, logrando que en el $85 \%$ de los proyectos presentados por las empresas participe una casa de estudio o centro de investigación. Estas alianzas constituyen una primera etapa que se podría denominar de acercamiento, para luego pasar a la segunda etapa que se le denominaría aportación a la productividad y desarrollo empresarial, estableciéndose una mínima participación de las universidades.

El rápido cambio tecnológico por el que atraviesa el mundo contemporáneo, con los grandes avances en las tecnologías de la información y las comunicaciones (TIC), así como la biotecnología y los nuevos materiales, plantean una serie de oportunidades y desafíos a la sociedad y a la estructura productiva de los distintos países a nivel mundial. De esta forma, es común escuchar que aquellos países que no logren adaptar para sí las transformaciones impulsadas por las nuevas tecnologías en la industria, agricultura, salud, medio ambiente, energía, educación y otros sectores, corren el riesgo fatal de quedarse a la zaga en términos de desarrollo y bienestar; y más aún en el caso particular de los países en desarrollo, de profundizar la llamada brecha tecnológica que los separa del mundo industrializado, de manera que es urgente el aporte efectivo de la universidad a la empresa y a la sociedad. 
Según el análisis de Romer se establece:

a) El cambio tecnológico definido como la mejora en la formulación de procesos para combinar las materias primas, es una fuerza esencial para el crecimiento económico que incentiva la continuidad en la acumulación de capital, ambos son factores que impulsan en gran parte del incremento en el producto obtenido por hora trabajada.

b) Los incentivos de mercado juegan un papel esencial en el proceso por el cual el nuevo conocimiento es usado para la producción de bienes y servicios esenciales.

c) El mercado de producción de nueva tecnología tiene características particulares que lo diferencian de las condiciones que rigen la oferta y la demanda del común de los bienes, cuya característica del cambio tecnológico es la de un bien público.

En la medida en que ha avanzado la evolución de las diferentes industrias, se hizo evidente que la obtención de nuevos conocimientos tecnológicos ha requerido ir más allá de la simple experiencia del personal involucrado en la producción. Surgen entonces los departamentos de investigación y desarrollo (I-D) en las modernas organizaciones empresariales, cuya operación al interior de estas se ha consolidado como factor fundamental en la generación de innovación para la organización.

En esta dirección la universidad tiene el rol esencial de transferir ciencia, tecnología e innovación a las empresas y particularmente impulsar la competitividad de las pymes. El propósito es establecer un mecanismo público-privado de trabajo conjunto y con cobertura nacional, que fomente un mejor desempeño competitivo de las pymes mediante la articulación interempresarial y la colaboración entre empresas e instituciones de apoyo, y promueva la colaboración público privada en el ámbito de las políticas de desarrollo productivo.

Que las universidades públicas respondan a su rol de desarrollo de la innovación y la tecnología, contribuyendo a elevar la productividad de las empresas y el bienestar de la sociedad peruana, así como propiciar que los profesionales universitarios que egresan sean altamente competitivos.

Que los perfiles de las carreras profesionales respondan a los cambios del entorno internacional y nacional a las necesidades de tecnología e innovación que experimentan las empresas en el país y que las grandes y pequeñas empresas que se desenvuelven en mercados altamente competitivos no tengan que proveerse de recursos humanos extranjeros.

En el vínculo universidad-empresa se expone su importancia por la imperante corriente de la globalización, representando para la universidad grandes desafíos, los cuales han sido planteados por los distintos organismos internacionales. La Unesco (Declaración mundial sobre educación superior: visión y acción 1998) reconoce la necesidad de ampliar la respuesta de la educación a las necesidades planteadas a través de redes, transferencia de tecnología y la creación de nuevos entornos pedagógicos, como la educación a distancia, el aprovechamiento de las tecnologías informatizadas, la capacidad de adaptar tecnología a las necesidades regionales y nacionales, seguir de cerca la evolución del conocimiento, fortalecer por medio de la cooperación internacional los intereses de todos los países, y modernizar el trabajo académico en lugar que este se transforme en un espacio virtual. Por consiguiente, la innovación también resulta ser un elemento clave para el desarrollo de las sociedades.

Tal situación ha dado lugar a que se revitalice el interés de la investigación sobre el papel de las instituciones de educación técnica y universitaria, en la calificación y recalificación permanente de los recursos humanos en todos los niveles de la escala empresarial y los criterios de su evaluación; pero también acerca de la función que los gobiernos centrales, estatales y locales tienen en la promoción y regulación de la actividad económica y empresarial, así como de sus vinculaciones con las instituciones educativas y de seguridad social como parte de una política industrial activa (Mungaray y Palacios). Sin embargo, especial interés han recibido las articulaciones entre dos actores: la universidad y la empresa. En muchos casos se recoge la experiencia de países como Estados Unidos y Japón, así como sus particulares modelos de articulación. Sin embargo, en México y en general en los países latinoamericanos, se han difundido y documentado poco.

En el caso de México existen proyectos de vinculación universidad-empresa que han generado entornos propicios para la creación de redes de cooperación entre los actores involucrados (empresarios, empleados, investigadores y estudiantes) -Dutrénit (1996, pp. 660-668), Coronado 
y Tapia (1996, pp. 825-833), Casas y Luna (1995)-, particularmente en las industrias de productos de medición (polímeros), químicas, alimentarias, textiles, entre otras. Desde la perspectiva regional, Baja California, por la dinámica que presenta, podría ser un ejemplo ilustrativo para la conformación de redes entre los diversos agentes económicos, sociales e instituciones públicas y privadas que promuevan las capacidades de innovación de las empresas locales y regionales, y sentarán las bases para la conformación de un sistema regional o local de innovación.

Independientemente de que el sistema de innovación sea abordado desde un ámbito nacional, regional, local o transnacional, la producción del conocimiento y la colaboración entre universidades y sector productivo son fundamentales. Hoy más que nunca las universidades, y en general todas las instituciones de educación superior, tienen un papel protagónico en el desarrollo de la innovación. Las universidades como productoras del conocimiento tienen un papel trascendental en el impulso de las capacidades tecnológicas que respondan de manera efectiva a las necesidades nacionales, regionales y locales. En esa dirección, en particular interesa para efectos de la presente investigación la vinculación entre dos actores de la innovación: la universidad y la empresa.

Lo anterior se deriva de las potencialidades que tienen las micro, pequeña y medianas empresas para generar el desarrollo regional, lo cual queda sustentado en el importante papel que juegan en los procesos de innovación y cambio tecnológico. Estudios recientes han mostrado que una gran parte de las innovaciones provienen de empresarios y de empleados de pequeños negocios, que al menos generan tantas innovaciones como las grandes empresas (Acs, 1992, pp. 38-44). En segundo lugar, por la capacidad que tienen para generar empleos, ya que sus procesos productivos se caracterizan por su intensidad en el factor trabajo. Finalmente, Durán (1995) y Mungaray (1995) señalan que en países como México, con importantes problemas de desequilibrio externo, la proliferación de pequeñas empresas puede ayudar a sustituir eficientemente importaciones por productos nacionales y así incrementar el multiplicador del gasto y fortalecer el crecimiento (Mungaray y Ramírez, 2000:68). Uno de los factores fundamentales para la innovación es el conocimiento en sus distintas modalidades, no solo el adquirido de manera formal en las instituciones y que se acredita mediante un documento oficial, sino también el adquirido de manera informal con la práctica diaria, el que viene de aprender "haciendo cosas" (López, 2000:67).

Aun cuando todavía prevalece la idea de que la ciencia pura es producida en las universidades en un ambiente académico, y la tecnología es producida en las empresas en un ambiente de producción, cada vez son más estrechas las relaciones entre las instituciones de la academia y las instituciones de la producción, debido al surgimiento de importantes desarrollos en los cuales participan instituciones de ambos perfiles (Ibíd.: 71). La interacción constituye un factor preponderante para el impulso de la actividad innovadora. Según Cimoli (2000), la actividad innovadora incluye todos los procesos mediante los cuales las empresas dominan y ponen en práctica diseños de productos y procesos manufacturados que son nuevos para ellos y crean valor.

La necesidad de armonizar el sector productivo con la educación en sus distintos niveles es una necesidad imperiosa e impostergable, en virtud de que solo a través de un esquema de vinculación el mercado laboral puede resultar favorecido y, en este sentido, ser congruentes las necesidades de servicios profesionales con las posibilidades de la oferta (Mungary y Ocegueda 1999). El crecimiento económico no solo debe descansar en la actividad exportadora o importadora, sino también en el desarrollo de ventajas competitivas construidas sobre la base de una mejor calidad en la infraestructura productiva y en una mejor formación y eficiencia del recurso humano. En suma, la educación deberá estar más articulada a la economía con el propósito de entender las nuevas formas de organización industrial y toda su base tecnológica. La necesidad de articular las relaciones entre los sistemas de educación y los sectores productivos, especialmente el industrial, requiere de un nuevo modelo de interacción entre las instituciones de educación superior con las empresas y las comunidades, que reconozcan las diferencias económicas y sociales existentes a la hora de planear la entrega de servicios de calidad de acuerdo con las asimétricas necesidades sociales, sectoriales y regionales existentes (Casas y Luna 1997).

La adecuada definición de los objetivos de aprendizaje en empresas competitivas y eficientes, respecto de los objetivos de aprendizaje en empresas con mayor necesidad de apoyo social, permitiría acelerar procesos de acercamiento a la 
cultura de la calidad y la organización en las micro y pequeñas empresas, con efectos similares a los que obtendrían si les fuera posible financiar y desarrollar por sí mismas la investigación y el desarrollo. Si la excelencia es el cumplimiento con las necesidades del entorno, entonces la excelencia universitaria supone una universidad promovida y sostenida participativamente por estudiantes, profesores, empresas y Estado.

\section{CONCLUSIONES}

1. La situación dinámica y de constante cambio que experimentan las organizaciones empresariales y académicas, ha dado lugar a que el rol del binomio universidad-empresa, se revitalice. Si bien las universidades cuentan con un elevado potencial de talento humano; estas deben encauzar sus esfuerzos de formación académico profesional en responder a las demandas exigidas por el mercado. Asimismo estas entidades deben ser dinámicas, adecuándose a los cambio en las tendencias laborales y a los cambios del entorno socio-económico.

2. La transferencia de investigación y tecnología eleva la importancia del capital humano, como pilar para fomentar la productividad en las empresas y la competitividad en el mercado inmerso en la corriente de la globalización.

3. Se establece la relación esencial del sector público-privado, cuya acción conjunta fomentará la creación de unidades o departamentos de Investigación y desarrollo $(\mathrm{I}+\mathrm{D})$ en las organizaciones empresariales orientado a elevar la productividad y la competitividad, y que impulse el mejoramiento de la calidad académico- profesional en las universidades, propiciando la empleabilidad.

\section{REFERENCIAS BIBLIOGRÁFICAS}

ACS, Z.J. 1992. "Small busines economics: a global perspective”. En Challenge, v. 35, num. 6.

Braun-Thürmann: Innovation.

Capdevielle, M. 2000. "Composición tecnológica de la industria manufacturera mexicana”. En Revista Mercado de Valores 50.

Carbaugh, Robert J, Economía internacional.

Casalet, M. 1994. "La formación profesional y técnica en México". En Revista Comercio Exterior 44 (8).
Casas, R. 2000. "El papel de las instituciones productoras de conocimiento en el desarrollo del sistema mexicano de innovación”. En Revista Mercado de Valores 50 (1).

Castro Díaz - Balart, F. 2002. Ciencia, innovación y futuro.

Centros de Información: Gremios empresariales, Concytec, Consejo Nacional de competitividad, Fincyt, etc.

Corona, J y Hernández, C. 2000. "Relación proveedor-usuario y flujos de información tecnológica en la industria mexicana”. En Revista Comercio Exterior 50 (9).

Coronado, M y Tapia, A. 1996. "Vinculación universidad- sector productivo: un estudio de la industria alimentaria”. En Revista Comercio Exterior 46 (10).

Departamento de investigación del Banco Interamericano de Desarrollo (BID). < http://www. iadb.org/res > .

Dutrénit, G. 1994. "Sistema Nacional de Innovación”. En Revista Comercio Exterior 44 (8).

Dutrénit, G. 1996. "La vinculación universidadempresa en un macro proyecto de polímeros. En Revista Comercio Exterior 46 (10).

Freeman C., 1974. Teoría económica de la innovación industrial.

Krugman Paul El retorno de la economía de la depresión, Economía Internacional.

Machado, Fernández M. - Gestión tecnológica para un salto en el desarrollo industrial.

Nouriel Roubini - Crisis Económica, como salimos de esta.

Pavón J. Y A. Hidalgo - Gestión e Innovación. Un enfoque estratégico.

Portal del Fondo Monetario Internacional. < http:// www.imf.org $>$.

Porter, Michael - Estrategia Competitiva.

Pottruck. David. Cómo generar ideas e innovación.

Roberts, E. Gestión de la innovación tecnológica.

Rodríguez C., Jesús. Tecnología e innovación.

Sagastegui Francisco. Ciencia, Tecnología, Innovación. 